УДК 796.011+612.6

\title{
ФУНКЦИОНАЛЬНОЕ СОСТОЯНИЕ ДЕТЕЙ 5-7 ЛЕТ С РАЗЛИЧНОЙ ВЫНОСЛИВОСТЬЮ ПРИ ЧРЕЗМЕРНО ИНТЕНСИВНОМ ИСПОЛЬЗОВАНИИ ЦИФРОВЫХ ТЕХНОЛОГИЙ
}

\author{
Герасимова Анастасия Альлеровна \\ доктор медицинских наук, профессор \\ Государственный университет управления \\ Криволапчук Иван Игоревич \\ научный сотрудник \\ Чернова Мария Борисовна \\ кандидат педагогических наук, доцент \\ Федеральное государственное \\ бюджетное научное учреждение \\ «Институт возрастной физиологии» \\ Российской академии образования \\ Савушкина Елена Васильевна \\ старший преподаватель; \\ УО «Гродненский государственный \\ университет имени Янки Купалы»
}

\footnotetext{
Аннотация. Сравнение дошкольников 6-7 лет с повышенными значениями модифицированного индекса информатизации условий жизнедеятельности, отличающихся по уровню выносливости, выявило значимые различия в отношении ключевых аспектов развития. Установлено, что дети с высокой общей и силовой выносливостью в условиях чрезмерно интенсивного использования цифровых технологий, характеризуются оптимальным функциональным, физическим и моторным развитием.

Ключевые слова: модифицированный индекс информатизации, общая и силовая выносливость, функциональное состояние, физическое и моторное развитие.
} 


\title{
FUNCTIONAL STATE OF 5-7 YEARS OLD CHILDREN WITH VARIOUS ENDURANCE WITH EXCESSIVE USE OF DIGITAL TECHNOLOGIES
}

\author{
Gerasimova Anastasia Allerovna \\ $\mathrm{PhD}$ (Doc. Med. Sci.), professor \\ State University of Management, Moscow, Russia \\ Krivolapchuk Ivan Igorevich \\ laboratory assistant-researcher \\ Chernova Maria Borisovna \\ $\mathrm{PhD}$ (Cand. Ped. Sci.), associate professor \\ The Federal State Budget Scientific Institution \\ «Institute of Developmental Physiology \\ of the Russian Academy of Education» \\ Savushkina Elena Vasil'evna \\ senior lecturer \\ Yanka Kupala State University of Grodno
}

\begin{abstract}
Comparison of 6-7-year-old preschoolers with increased modified informatization index of living conditions and different levels of endurance revealed significant differences in the key aspects of development. Children with high general and strength endurance under conditions of excessive use of digital technologies were found to have optimal functional, physical and motor development.
\end{abstract}

Key words: modified informatization index, general and strength endurance, functional state, physical and motor development.

Введение. Сегодня проводится большое количество научных исследований, касающихся оздоровительной роли физической активности и двигательной подготовленности детей в условиях интенсивного использования цифровых технологий $[11,16,5,2,3]$. В ряде работ выявлена отрицательная взаимосвязь между чрезмерно высоким объемом цифровой нагрузки в режиме дня, с одной стороны, физической активностью, морфофункциональным, моторным развитием и здоровьем детей, с другой $[13,4,19$, $18,16,8,1,7]$. Вместе с тем, несмотря на имеющиеся данные, существует ряд нерешенных проблем, связанных с оценкой влияния цифровых технологий на 


\section{МОДЕРНИЗАЦИЯ СОВРЕМЕННОГО ОБРАЗОВАНИЯ: АНАЛИЗ ОПЬТА И ТЕНДЕНЦИЙ}

функциональное состояние, физическое и моторное развитие детей дошкольного возраста с учетом уровня проявления кондиционных физических способностей. Особый интерес в этом отношении представляет выносливость, как многофакторная физическая способность, в значительной степени определяющая адаптационные возможности и работоспособность человека в процессе индивидуального развития.

Цель исследования - выявить особенности функционального состояния детей 5-7 лет с разным уровнем развития общей и силовой выносливости в условиях чрезмерно интенсивного использования цифровых технологий.

Методологические подходы. В исследовании приняли участие практически здоровые дети 6-7 лет $(\mathrm{n}>2100)$. Организация исследований основывалась на требованиях Хельсинской Декларации.

Для выявления особенностей применения детьми цифровых технологий в повседневной жизни проводился хронометраж разных видов деятельности, опрос родителей и воспитателей детских дошкольных учреждений. Все виды деятельности, связанные с использованием цифровых технологий, суммировались. Посредством анализа специального дневника определяли продолжительность ночного и дневного сна. На этой основе рассчитывали модифицированный индекс информатизации (Imod) [10]. Данный индекс равен отношению количества часов использования цифровых технологий в течение суток к общему времени бодрствования, выраженному в процентах [1].

Регистрировали частоту сердечных сокращений (ЧСС), систолическое (СД) и диастолическое (ДД) давление крови по Короткову в соответствии с рекомендациями ВО3. На основании этих измерений по общеизвестным формулам определяли среднее давление (САД), двойное произведение (ДП), индекс Мызникова (ИМ), вегетативный индекс Кердо (ВИК) и индекс функциональных изменений (ИФИ). Измеряли окружность грудной клетки (ОГК), рост и массу тела. Полученные результаты использовали для расчета индекса массы тела (ИМТ). Батарея моторных тестов включала добротные контрольные упражнения для оценки двигательной подготовленности: бег 30 м, наклон вперёд, челночный бег $3 \times 10$ м, поднимание туловища из положения лёжа на спине за минуту, прыжок в длину с места, шестиминутный бег.

Рассчитывали основные статистические характеристики ряда измерений. На основе использования параметрических и непараметрических критериев проверяли статистические гипотезы. Использование 


\section{МОДЕРНИЗАЦИЯ СОВРЕМЕННОГО ОБРАЗОВАНИЯ: АНАЛИЗ ОПЫТА И ТЕНДЕНЦИЙ}

модифицированного индекса информатизации условий жизнедеятельности позволило распределить дошкольников на 5 функциональных классов, отражающих интенсивность использования цифровых технологий: низкий $(<\mathrm{M}-1,0 \sigma)$, ниже среднего (от $\mathrm{M}-1,0 \sigma$ до $\mathrm{M}-0,5 \sigma)$, средний $(\mathrm{M} \pm 0,5 \sigma)$ выше среднего (от $\mathrm{M}+0,5 \sigma$ до $\mathrm{M}+1,0 \sigma)$ и высокий $(>\mathrm{M}+1,0 \sigma)$ [1]. В дальнейшем исследовании принимали участие дошкольники, имеющие высокий уровень информатизации условий жизнедеятельности (n>254). Полученную выборку детей, характеризующихся интенсивным использованием цифровых технологий в повседневной жизни, на основе описанного выше способа дифференцировали по пяти 5 уровням развития выносливости. Сравнивались дошкольники с высоким и низким уровнями развития общей (шестиминутный бег) и силовой (поднимание туловища) выносливости.

Результаты исследования. Анализ особенностей функционального состояния организма, физического и моторного развития дошкольников 6-7 лет с повышенным индексом информатизации условий жизнедеятельности выявил различия, обусловленные уровнем двигательной подготовленности.

Сравнение двигательной подготовленности детей, отличающихся по результатам выполнения шестиминутного бега, позволило установить, что выносливые дошкольники по сравнению детьми, демонстрирующими «слабые» результаты выполнения данного контрольного упражнения, характеризовались относительно низкими ( $<0,05-0,001)$ значениями ряда вегетативных показателей функционального состояния и физического развития: СД, ДД, ЧСС, ВИК, ДП, ИМ, ИФИ, масса тела и ИМТ. Выявлены также различия по уровню развития скоростных, скоростно-силовых и координационных способностей, гибкости и силовой выносливости. Мальчики и девочки с высокой общей выносливостью опережали сверстников с низкими результатами шестиминутного бега по всем используемым моторным тестам $(\mathrm{p}<0,001)$. По средним значениям Imod межгрупповые различия не выявлены.

Сходные результаты были получены в отношение силовой выносливости. Дети 6-7 лет с высоким Imod, подразделенные на группы по результатам выполнения теста «поднимание туловища», существенно отличались по показателям функционального состояния и физического развития. Различия ( $<<0,05-0,01)$ касались средних значений СД, САД, ЧСС, ВИК, ИМ, ИФИ, ИМТ. В отношение величины Imod различия отсутствовали. 
У них более высокий уровень развития силовой выносливости сочетался с более высокими уровнями проявления скоростных, скоростно-силовых и координационных способностей, общей выносливости.

Таким образом, дошкольники с высокой общей и силовой выносливостью, в целом характеризовались «лёгким» сдвигом вегетативного баланса в сторону преобладания активности парасимпатического отдела вегетативной нервной системы, большей экономичностью функционирования системы кровообращения и большими адаптационными возможностями организма по сравнению со сверстниками с низкими результатами выполнения шестиминутного бега и теста «поднимание туловища». У детей с высоким уровнем общей выносливости отмечалось также сбалансированное физическое развитие. Полученные результаты в целом соответствуют данным о возможном ухудшении ФС организма, снижении физической активности и двигательной подготовленности детей в условиях чрезмерно интенсивного использования цифровых технологий $[16,6,3,8]$.

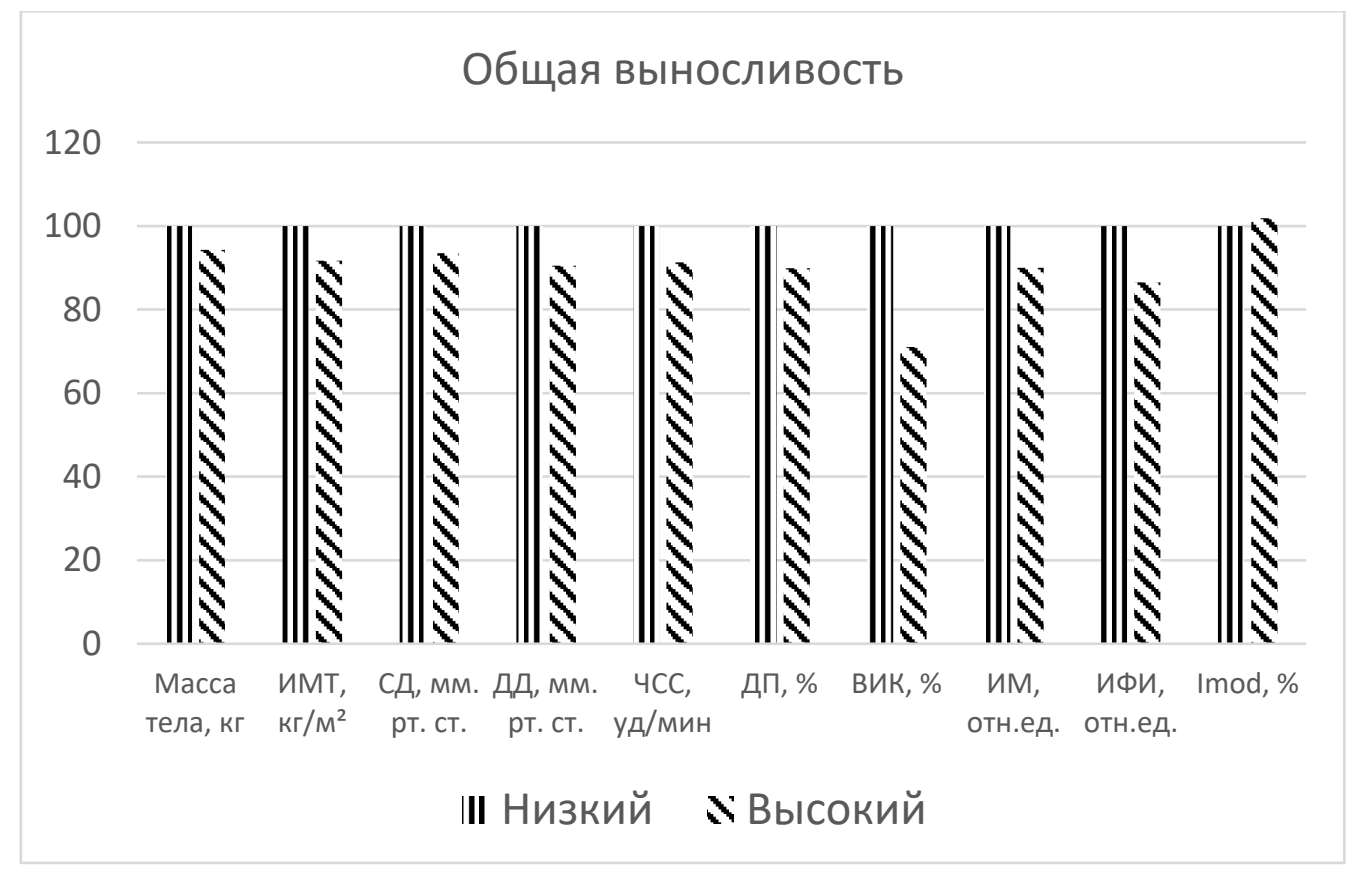

\section{Рис. 1. Особенности ФС детей 6-7 лет с высоким и низким уровнем развития выносливости (шестиминутный бег) при интенсивном использовании цифровых технологий}

Примечание. За 100 \% приняты средние величины показателей у дошкольников с низким уровнем двигательной подготовленности. 
Материалы исследования хорошо согласуются с представлением, что одним из основных факторов, способствующих возникновению проблем, связанных с неблагоприятным функциональным состоянием и здоровьем детей в условиях интенсивной информатизации, является уменьшение объема физической активности и снижение уровня развития кондиционных двигательных способностей $[13,9,6,14]$.

Данные о том, что дети 6-7 лет с высоким уровнем развития общей и силовой выносливости в условиях чрезмерно интенсивного использования цифровых технологий в повседневной жизни характеризуются хорошей двигательной подготовленностью, оптимальным физическим развитием и повышенными функциональными возможностями организма, в целом соответствуют выводам других работ $[19,6,15,8]$.

В ряде исследований зарубежных авторов, показано, что объем экранного времени менее 2 часов в сутки можно рассматривать как приемлемый для детей старшего дошкольного возраста, а превышающий 4 часа - как неоправданно высокий $[17,19]$. Установлено, что увеличение до 2-х и более часов в день затрат времени на цифровые технологии приводит к снижению физической активности $[12,19]$.

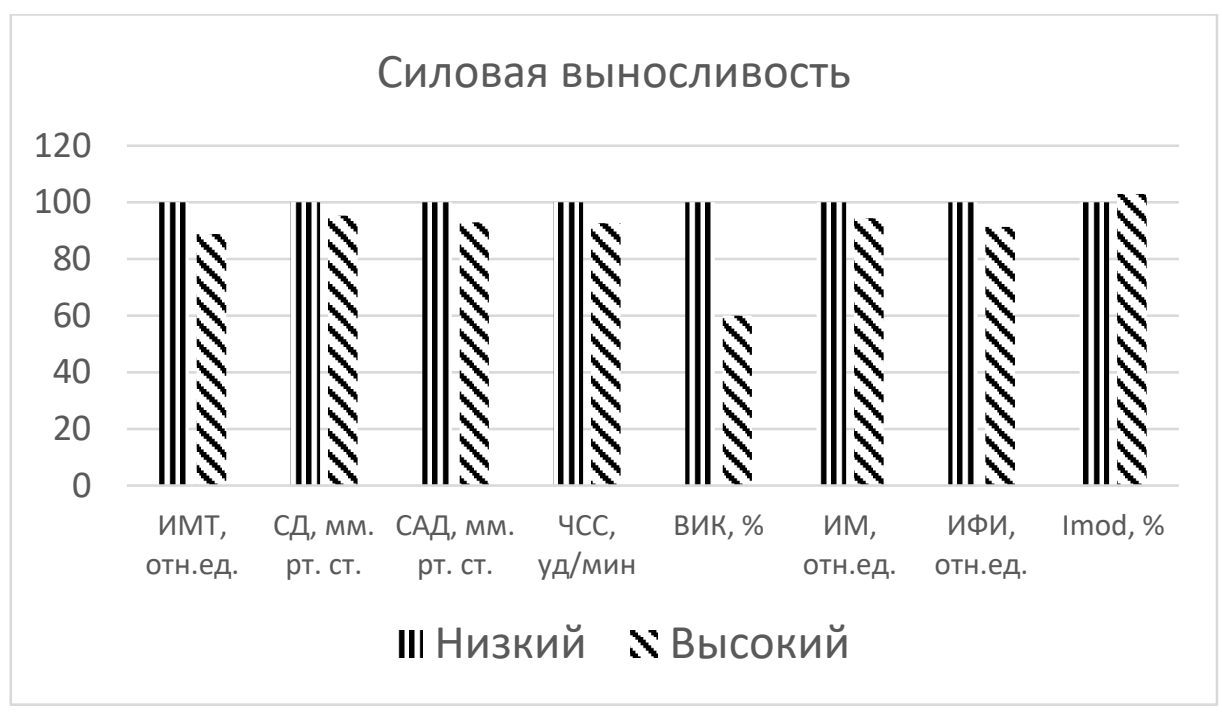

Рис. 2. Особенности ФС детей 6-7 лет с высоким и низким уровнем развития выносливости (поднимание туловища из положения лежа на спине) при интенсивном использовании цифровых технологий

Примечание. За 100 \% приняты средние величины показателей у дошкольников с низким уровнем двигательной подготовленности. 


\section{МОДЕРНИЗАЦИЯ СОВРЕМЕННОГО ОБРАЗОВАНИЯ: АНАЛИЗ ОПЬТА И ТЕНДЕНЦИЙ}

Наши данные показывают, что среди детей с высокой степенью информатизации условий жизнедеятельности общее экранное время намного превышает границы оптимального диапазона, рекомендованного для дошкольников. Имеется ряд работ, свидетельствующих об отрицательной связи экранного времени с развитием выносливости у детей $[17,15,3]$. Установлено, что время использования наиболее популярных социальных сетей отрицательно коррелирует с уровнем общей выносливости [17]. Выявлено, что наблюдаемое в ходе онтогенеза увеличение экранного времени, связано с более низким уровнем общей выносливости и физической активности детей [13]. Показано, что каждый час экранного времени в день снижает вероятность достижения нормативного уровня развития общей выносливости [6].

Заключение. Сравнение дошкольников 6-7 лет с повышенными значениями модифицированного индекса информатизации условий жизнедеятельности, отличающихся по уровню выносливости, выявило значимые различия в отношение ключевых аспектов развития. Установлено, что дети с высокой общей и силовой выносливостью в условиях чрезмерно интенсивного использования цифровых технологий, характеризуются оптимальным функциональным, физическим и моторным развитием.

C учетом полученных результатов необходимо отметить, что повышение двигательной подготовленности детей на основе направленного применения средств, методов и технологий физического воспитания в режиме дня может способствовать повышению устойчивости к повышенным информационным нагрузкам, а также упорядочению времени использования цифровых технологий в свободное время.

Выявленные различия необходимо учитывать при организации процесса физического воспитания детей дошкольного возраста в условиях повышенных информационных нагрузок. Исследование выполнено при финансовой поддержке РФФИ (грант № 20-013-00119). 


\section{МОДЕРНИЗАЦИЯ СОВРЕМЕННОГО ОБРАЗОВАНИЯ: АНАЛИЗ ОПЫТА И ТЕНДЕНЦИЙ}

\section{Список литературы}

1. Криволапчук, И.А. Функциональное развитие детей 6-7 лет с разным уровнем информатизации условий жизнедеятельности / И.А. Криволапчук, М.Б. Чернова, А.А. Герасимова - Текст: непосредственный // Сибирский педагогический журнал. - 2020. - №. 5. - С. 121-135.

2. Криволапчук, И.А. Влияние средств информатизации на физическую активность детей школьного возраста (обзор зарубежных исследований) / И.А Криволапчук, М.Б. Чернова, И.И. Криволапчук - Текст: непосредственный // Новые исследования. - 2019 - №2. - С. 5-14.

3. Cabanas-Sánchez, V. Associations of total sedentary time, screen time and non-screen sedentary time with adiposity and physical fitness in youth: the mediating effect of physical activity / V. Cabanas-Sánchez, D. Martínez-Gómez, I. Esteban-Cornejo, A. Pérez-Bey, J. Castro Piñero, O.L. Veiga // J Sports Sci. 2019. - Vol. 37. - № 8. - P. 839-849.

4. Carson, V. Physical activity and sedentary behavior across three timepoints and associations with socialskills in early childhood / V. Carson, E.Y. Lee, K.D. Hesketh, S. Hunter, N. Kuzik, M. Predy, R.E. Rhodes, C.M. Rinaldi, J.C. Spence, T. Hinkley // BMC Public Health. - 2019. - Vol. 19, №1. - P. 27-34. doi: 10.1186/s12889-018-6381-x.

5. Emm-Collison, L.G. Striking a Balance: Physical Activity, ScreenViewing and Homework during the Transition to Secondary School / L.G. EmmCollison, S. Lewis, T. Reid, J. Matthews, S.J. Sebire, J.L. Thompson, R. Salway, R. Jago // Int J Environ Res Public Health. - 2019. - Vol. 16. - №17. - P. 3174. doi: 10.3390/ijerph16173174

6. Hardy, L.L. Association Between Sitting, Screen Time, Fitness Domains, and Fundamental Motor Skills inChildren Aged 5-16 Years: Cross-Sectional Population Study / L.L. Hardy, D. Ding, L.R. Peralta, S. Mihrshahi, D. Merom // J Phys Act Health. - 2018. - Vol. 15. - № 12. - P. 933-940. doi: 10.1123/jpah.20170620.

7. Holton, V. A Nationwide Study of Myopia in Taiwanese School Children: Family, Activity, and School-Related Factors / V. Holton, J.E. Hinterlong, C.Y. Tsai, J.C. Tsai, J.S. Wu, Y.M. Liou // J Sch Nurs. - 2021. - Vol. 37(2). P. 117-127. doi: 10.1177/1059840519850619.

8. Janssen, X. Associations of screen time, sedentary time and physical activity with sleep in under 5s: A systematic review and meta-analysis / X. Janssen, 
A. Martin, A.R. Hughes, C.M. Hill, G. Kotronoulas, K.R. Hesketh // Sleep Med Rev. - 2019. - Vol. 49. - P. 101-126. https://doi.org/10.1016/j.smrv.2019.101226

9. Kremer, P. Physical activity, leisure-time screen use and depression among children and young adolescents / P. Kremer, C. Elshaug, E. Leslie, J.W. Toumbourou, G.C. Patton, J. Williams // J Sci Med Sport. - 2014. - Vol. 17, № 2. - P. 183-187. doi: 10.1016/j.jsams.2013.03.012.

10. Krivolapchuk, I.A. Modified Informatization Index of Children's Life / I.A. Krivolapchuk, M.B. Chernova, A.A. Gerasimova, V.P. Chicherin, V.V. Myshyakov // Smart Technologies" for Society, State and Economy / Ed. Elena G. Popkova, Bruno S. Sergi. - 2020, Springer Nature, pp. 245-247.

11. Lepp, A. The relationship between cell phone use, physical and sedentary activity, and cardiorespiratory fitness in a sample of U.S. college students/ A. Lepp, J. Barkley, G. Sanders, M. Rebold, P. Gates // International Journal of Behavioral Nutrition and Physical Activity. - 2013. - Vol. 10. - P. 79-87. DOI: 10.1186/14795868-10-79

12. Melkevik, O. Is spending time in screen-based sedentary behaviors associated with less physical activity: a cross national investigation/ O. Melkevik, T. Torsheim, R.J. Iannotti, B. Wold //Int J Behav Nutr Phys Act. - 2010. - Vol. 7, P. 46. doi: 10.1186/1479-5868-7-46.

13. Mitchell, J.A. Screen-based sedentary behavior and cardiorespiratory fitness from age 11 to 13 / J.A. Mitchell, R.R. Pate, S.N. Blair // Med Sci Sports Exerc. - 2012. - Vol. 44, № 7. -P. 1302-1309. doi: 10.1249/MSS. 0b013e318247cd73.

14. Mustafaoğlu, R. The negative effects of digital technology usage on children's development and health. Addicta: The Turkish / R. Mustafaoğlu, E. Zirek, Z. Yasacı, A. Razak Özdinçler // Journal on Addictions. - 2018. - № 5. P. 227-247. http://dx.doi.org/10.15805/addicta.2018.5.2.0051

15. Pfledderer, C.D. Association between Access to Electronic Devices in the Home Environment and Cardiorespiratory Fitness in Children / C.D. Pfledderer, R.D. Burns, T.A. Brusseau // Children (Basel). - 2019. - Vol. 6, № 1. - P. 8. doi: 10.3390/children6010008.

16. Potter, M. Behavior Tracking and 3-Year Longitudinal Associations Between Physical Activity, Screen Time, and Fitness Among Young Children / M. Potter, J.C. Spence, N. Boulé J.A. Stearns, V. Carson // Pediatr Exerc Sci. 2018. Vol. 30, № 1. - P. 132-141. doi: 10.1123/pes.2016-0239. 
17. Sandercock, G.R. Independence of physical activity and screen time as predictors of cardiorespiratory fitness in youth / G.R.Sandercock, A.A. Ogunleye // Pediatr. Res. - 2013. - Vol. 73. - P. 692-697.

18. Straker, L. Conflicting Guidelines on Young Children's Screen Time and Use of Digital Technology Create Policy and Practice Dilemmas / L. Straker, J. Zabatiero, S. Danby, K. Thorpe, S. Edwards // J Pediatr. - 2018. - Vol. 202. P. 300-303. doi: 10.1016/j.jpeds.2018.07.019.

19. Tremblay, M.S. Canadian 24-hour movement guidelines for children and youth: an integration of physical activity, sedentary behaviour, and sleep / M.S.Tremblay, V. Carson, J-P. Chaput, S. Connor Gorber, T. Dinh, M. Duggan // Appl Physiol Nutr Metab. - 2016. - Vol. 41(6 Suppl 3). - S. 311-327. DOI: 10.1139/apnm-2016-0151

(С) А.А. Герасимова, И.И. Криволапчук, М.Б. Чернова, Е.В. Савушкина, 2021 